\title{
Effectiveness of Peer-supervision on Paediatric Fever Treatment among Registered Private Drug Sellers in East-Central Uganda: An Interrupted Time Series Analysis
}

\author{
Arthur Bagonza ${ }^{1}$, Freddy Kitutu ${ }^{1}$, Stefan Peterson ${ }^{2}$, Andreas Mårtensson $^{2}$, Milton Mutto ${ }^{3}$, \\ Phyllis Awor ${ }^{1}$, David Mukanga ${ }^{4}$, and Henry Wamani ${ }^{1}$ \\ ${ }^{1}$ Makerere University College of Health Sciences \\ ${ }^{2}$ Uppsala University \\ ${ }^{3}$ Makerere University School of Public Health \\ ${ }^{4}$ Bill \& Melinda Gates Foundation
}

June 18, 2020

\begin{abstract}
Rationale, aims and objectives: Appropriate treatment of paediatric fever in rural areas remains a challenge and may be partly due to inadequate supervision of licensed drug sellers. This study assessed the effectiveness of peer-supervision among drug sellers on appropriate treatment of pneumonia symptoms, uncomplicated malaria and non-bloody diarrhoea among children less than five years of age in the intervention (Luuka) and comparison (Buyende) districts, in East-Central Uganda. Methods: Data on pneumonia symptoms, uncomplicated malaria and non-bloody diarrhoea among children less than five years of age was abstracted from drug shop sick child registers over a 12-month period; six months before and six months after introduction of peer-supervision. Interrupted time series was applied to determine the effectiveness of the peer-supervision intervention on appropriate treatment of pneumonia, uncomplicated malaria and non-bloody diarrhoea among children less than five years of age attending drug shops in East Central Uganda. Results: The proportion of children treated appropriately for pneumonia symptoms was $10.84 \%(\mathrm{P}<0.05, \mathrm{CI}=[1.75,19.9])$ higher, for uncomplicated malaria was $1.46 \%(\mathrm{P}=0.79, \mathrm{CI}=[-10.43$, 13.36]) higher, and for non-bloody diarrhoea was $4.00 \%(\mathrm{p}<0.05, \mathrm{CI}=[-7.95,-0.13])$ lower in the intervention district than the comparison district, respectively. Post-intervention trend results showed an increase of $1.21 \%(\mathrm{p}=0.008, \mathrm{CI}=[0.36,2.05])$ in the proportion appropriately treated for pneumonia symptoms, no difference in appropriate treatment for uncomplicated malaria, and a reduction of $1 \%(\mathrm{p}<0.06, \mathrm{CI}=[-1.95,0.02])$ in the proportion of children appropriately treated for nonbloody diarrhoea, respectively. Conclusions: Peer-supervision increased the proportion of children less than five years of age that received appropriate treatment for pneumonia symptoms but not for uncomplicated malaria and non-bloody diarrhoea. Implementation of community level interventions to improve paediatric fever management should consider including peersupervision among drug sellers.
\end{abstract}

\section{Methods:}

Data on pneumonia symptoms, uncomplicated malaria and non-bloody diarrhoea among children less than five years of age was abstracted from drug shop sick child registers over a 12-month period; six months before and six months after introduction of peer-supervision. Interrupted time series was applied to determine the effectiveness of the peer-supervision intervention on appropriate treatment of pneumonia, uncomplicated malaria and non-bloody diarrhoea among children less than five years of age attending drug shops in East Central Uganda.

\section{Results:}


The proportion of children treated appropriately for pneumonia symptoms was $10.84 \%(\mathrm{P}<0.05, \mathrm{CI}=$ $[1.75,19.9])$ higher, for uncomplicated malaria was $1.46 \%(\mathrm{P}=0.79, \mathrm{CI}=[-10.43,13.36])$ higher, and for non-bloody diarrhoea was $4.00 \%$ ( $\mathrm{p}<0.05, \mathrm{CI}=[-7.95,-0.13])$ lower in the intervention district than the comparison district, respectively.

Post-intervention trend results showed an increase of $1.21 \%(\mathrm{p}=0.008, \mathrm{CI}=[0.36,2.05])$ in the proportion appropriately treated for pneumonia symptoms, no difference in appropriate treatment for uncomplicated malaria, and a reduction of $1 \%(\mathrm{p}<0.06, \mathrm{CI}=[-1.95,0.02])$ in the proportion of children appropriately treated for non-bloody diarrhoea, respectively.

\section{Conclusions:}

Peer-supervision increased the proportion of children less than five years of age that received appropriate treatment for pneumonia symptoms but not for uncomplicated malaria and non-bloody diarrhoea. Implementation of community level interventions to improve paediatric fever management should consider including peer-supervision among drug sellers.

\section{Keywords}

Appropriate treatment, interrupted time series, peer-supervision, childhood illnesses, Uganda

\section{INTRODUCTION}

Acute febrile illness is a major cause of morbidity and mortality among children less than five years of age in low and middle income countries ${ }^{1-3}$. In 2018, the World Health Organization (WHO) reported that acute febrile illnesses due to malaria and diarrhoeal diseases caused approximately 272,000 and 525,000 deaths among children less than five years of age, respectively ${ }^{4,5}$. An additional 808,694 deaths of children less than five years were caused by pneumonia in $2019^{6}$. Majority of the deaths due to malaria, diarrhoea and pneumonia occur in Africa ${ }^{7,8}$.

Uganda is one of six countries that accounted for more than half of all malaria cases worldwide in $2019^{5}$. According to the Ministry of Health (MoH) Uganda, in 2019, pneumonia, malaria and diarrhoea combined accounted for over $30 \%$ mortalities in children less than five years of age making the three febrile illnesses the leading causes of death among this age group ${ }^{9}$.

In a bid to curtail common childhood illnesses among this vulnerable age group, in 2010, the WHO and the United Nations Children's Fund (UNICEF) recommended the provision of integrated community case management(ICCM) at community level ${ }^{10,11}$. The ICCM is a strategy focused on enhancing equity of public health services by providing timely and effective treatment of malaria, pneumonia and diarrhoea to populations with limited access to facility-based health care. In Uganda, ICCM was extended to include private health providers comprised mainly of drug shops targeting those who offer care to children less than five years of age.

Drug shops are small medicine outlets authorised to sell class-C drugs over- the- counter(OTC) that do not require prescription, used for treating minor and self-limiting conditions. Class $\mathrm{C}$ drugs are regarded as relatively safe drugs. They may also sell cosmetics and sundries deemed fit for sale by the Uganda National Drug Authority (NDA) - the medicine regulatory agency ${ }^{12}$.

More than half $(59 \%)$ of the children less than five years of age with febrile illness first seek care from private health providers because of physical in access to, and sometimes health system challenges in public health facilities ${ }^{13,14}$. Despite receiving iCCM training and government inspection, inappropriate treatment of febrile children persists among drug sellers in the small medicine outlets ${ }^{15}$.

The national professional guidelines for licensing, renewal and new licenses for class $\mathrm{C}$ drug shops in Uganda stipulate that licensed drug sellers shall be responsible for supervising drug shops which they own and or operate ${ }^{16}$. Relatedly, inspection of drug shops is carried out by the NDA on behalf of MoH. The NDA does periodic inspection through zonal inspectors. The zonal inspectors collaborate with District Drug Inspectors 
(DDIs) who derive their inspection mandate from the District Health Officer (DHO) at district level. Other officers under the DHO's office are illustrated in Fig. 1

Countrywide, DDIs are required to visit every drug shop in their district once every month ${ }^{17}$. However, inspection of drug shops by district drug inspectors and self-supervision as is practised by licensed drug sellers in rural settings seem not to address the inappropriate treatment of febrile illnesses in children less than five years.

Aim of the study

This study assessed the effectiveness of peer-supervision among drug sellers on appropriate treatment of pneumonia symptoms, uncomplicated malaria, and non-bloody diarrhoea among children less than five years of age in an intervention (Luuka) and comparison (Buyende) district in East-Central Uganda.

\section{Methods}

\subsection{Design of the study}

This study employed a quasi-experimental study design ${ }^{18-20}$ that assessed the proportion of children aged five years or less appropriately treated for pneumonia symptoms, uncomplicated malaria and non-bloody diarrhoea. The pre-intervention period was between May and October 2016 while peer supervision was carried out from November 2016 to May 2017.

\subsection{Setting of the study}

Intervention district with peer supervision

Investigators of this study used peer-supervision to augment the current self-supervision being practiced by drug sellers. Peer-supervision was aimed at supporting inspection of drug sellers by DDIs since evidence shows that inspection coupled with supervision improves quality of care $^{21}$. Peer-supervision is a type of supervision where supervisees encourage and enhance learning and development as peers. Peers are people of similar hierarchical status or who perceive themselves as equal ${ }^{22}$. Since peer supervision had been successful elsewhere ${ }^{23-25}$, it was envisaged that learning and development facilitated by peers would improve drug seller treatment of febrile illnesses in children under five years of age.

The intervention was carried out in Luuka district which has a total population of 238,020 persons $^{26}$. Presently, the district has no hospital, has one health centre level IV, six health centres level III and 16 health centres level II.

Peer-supervisors in the intervention district were chosen according to proposed criteria. The criteria involved peer supervisors being democratically chosen by drug sellers from that particular sub-county by show of hands. Having higher academic qualifications compared to other drug sellers was the second criteria. In the event that the peer supervisors chosen had similar qualifications, the person with the highest number of votes became the peer supervisor for that sub-county. Each sub-county in the intervention district had a peer supervisor. The peer supervisors underwent refresher iCCM training in October 2016. The refresher training was carried out by the investigators of the study and was based on ICCM guidelines by WHO/UNICEF adopted by the Ministry of Health Uganda ${ }^{27}$. The training lasted a total of three days for each supervisor in each sub-county. Peer supervisors were deemed fit for supervision if they could explain to the trainers what constituted appropriate treatment including recognizing danger signs in children less than five years with pneumonia symptoms, uncomplicated malaria and non-bloody diarrhoea. The training was supplemented by clearly defining roles for peer supervisors. These roles included; instructing and monitoring drug sellers on how to correctly fill sick child registers. In addition, peer supervisors were taught how to counsel drug sellers that were not giving appropriate treatment to children under five years of age. Peer supervisors were also mandated to cross check with drug sellers whether the respiratory timers and brand of RDTs being used were the recommended ones by the ministry of health.

More so, peer supervisors were taught how to be role models in their course of supervision by advising 
drug sellers to adhere to treatment guidelines. Peer supervisors were instructed to adhere to the highest form of privacy, professionalism, integrity and empathy. In all sub-counties of the intervention district, peer supervisors were tasked to work with an active district drug shop association where drug sellers met every month particularly, to attend continuous medical education organized by the drug shop association secretariat.

Peer supervisors were then provided with supervision checklists were they were asked to summarise treatment given to children by drug sellers on a monthly basis. The summarised information from the checklists was used by investigators to corroborate with information of drug shop sick child registers filled in by drug sellers every month. This was done to ensure accuracy of data filled in by drug sellers and data collected by peer supervisors. To ease the work of peer supervisors, the peer supervisors were also provided with summary extracts from iCCM treatment and referral algorithms ${ }^{27}$.

In line with guidelines for data collectors of the school of public health, Makerere, Uganda, every peer supervisor was given a safari day allowance of 80,000 Uganda shillings (equivalent to USD 22 at an exchange rate of USD 1 equal to 3,700 Uganda shillings). A safari day allowance is paid when a data collector travels within Uganda for a period of six hours or more and returns the same day. It is paid to cater for lunch, transport and other incidentals. The assumption was that each peer supervisor would visit all drug sellers within the sub-county every month and that supervision visits would not exceed one day.

The aim of peer supervision was to strengthen the existing health system by supporting the district local government. During peer supervision, the peer supervisors worked directly under the office of the DDI who carried on his inspection role as usual.

Comparison district

Buyende district has a population of 323,067 persons $^{26}$. The district has one health centre level IV, six health centres level III, eleven health centres level II and approximately 503 village health team members. In both districts, there was at least one drug shop in every village. The East-Central region where these two districts are located has a very high under-five mortality ranging between 73 to 90 per 1000 live births ${ }^{28}$.

\subsection{Characteristics of participants and description of materials}

In both districts, there were 135 registered drugs shops (Luuka 60 and Buyende 75 ) operated by nursing assistants, enrolled and comprehensive nurses, midwives and clinical officers. By law, drug shops in Uganda are authorised to sell class-C drugs (over the counter) that do not require prescription, used for treating minor and self-limiting conditions and are relatively safe. However, with the introduction of the iCCM strategy in the private sector, drug sellers were allowed to prescribe and dispense drugs for malaria (artemisinin combination therapies), pneumonia (amoxicillin) and diarrhoea (a combination of zinc and ORS) for children. This study was conducted among registered drug shops.

Before introduction of peer-supervision, both districts received training on how to treat children less than five years presenting with symptoms of pneumonia, uncomplicated malaria and non-bloody diarrhoea based on standard treatment guidelines developed by UNICEF, $\mathrm{MoH}$ and $\mathrm{WHO}^{27}$. The training was conducted between May 2015 and May 2016 by the Clinton Health Access Initiative (CHAI). The period between May 2016 and October 2016 was the period before peer-supervision was introduced.

\subsection{Data collection}

Socio-demographic data was collected from drug sellers using a questionnaire. Data on number of government inspection visits per drug shop was collected on a monthly basis from both districts from drug sellers when information on appropriate febrile treatment was being collected. Only data on number of peer-supervision visits was collected from the intervention district after introduction of peer supervision. Other data collected from drug shops included: age, gender, and qualifications of drug seller. In addition, prescription and treatment data of the under-five children who attended the drug shops was extracted from sick child registers 
line by line as is, to ensure accurate data capture. This data included name of the child, age, gender, care giver name, duration of symptoms, danger signs, respiratory rate (breaths per minute), fast breathing, RDT results as well as any other symptoms. Names of the children and care givers were given unique identifiers and anonymised during data management, analysis and report writing. Data was collected from the intervention and comparison districts between June 2016 and May 2017.

\section{Outcome variable}

The outcome variable for the study was appropriate treatment-defined as a drug seller prescribing and dispensing age appropriate medication to children less than five years old with symptoms of pneumonia, uncomplicated malaria and non-bloody diarrhoea in the right dose, frequency and duration for the right indication as per the standard iCCM treatment guidelines ${ }^{27}$.

\subsection{Data analysis}

To assess the effectiveness of peer supervision among drug sellers on appropriate treatment of children less than five years of age with pneumonia symptoms, uncomplicated malaria and non-bloody diarrhoea, interrupted time series for multiple group analysis was conducted ${ }^{29}$. The ITSA method was the preferred choice for this this study because it was not possible to randomise peer supervision (the intervention $)^{30}$. In addition we wanted to cater for policy shifts if they occurred during the study period-a major strength of using interrupted time series ${ }^{31}$. Consideration was made in using interrupted time series since the study had six data points before and six data points after introduction of peer supervision which are considered a minimum for reliable results ${ }^{32,33}$.

The mean with the corresponding standard deviation and median number of visits by DDIs and peer supervisors was computed. Then, the percentage of appropriately treated children by drug seller by month by district was calculated by dividing the total number of appropriately treated children for a given childhood illness by the total number of children presenting with symptoms of that particular illness multiplied by 100 . Results were then aggregated at district level by month covering both the pre-intervention and intervention period and used to show the trend of appropriate treatment in both districts for both periods.

This was followed by fitting the multiple group ITSA model (Equation 1). In the final step, the CumbyHuizinga test was used to test for autocorrelation. The model was re-run by specifying the lag order accounting for autocorrelation. Data from the sick child registers was entered in Epi Data (www.epidata.dk), exported to excel for cleaning and coding. Data analysis was done using STATA version 15 (Stata Corp, College Station, TX, 2015).The ITSA model is as follows;

$y_{t}=\beta_{0}+\beta_{1} T_{t}+\beta_{2} X_{t}+\beta_{3} X_{t} T_{t}+\beta_{4} Z+\beta_{5} Z T_{t}+\beta_{6} Z X_{t}+\beta_{7} Z X_{t} T_{t}+\epsilon_{t}$. (Equation 1)

The names and definitions of all variables used in this model are listed in table 1.

The ITSA uses ordinary least squares (OLS) approaches to estimate the effects of the intervention (peer supervision) on appropriate treatment for multiple treatment (intervention) periods. The ITSA model accounts for auto correlation among equally spaced observation data by comparison for the lag order for which auto correlation is assumed to be present. The estimated coefficients from the model together with their standard errors are reported.

\section{RESULTS}

\subsection{Descriptive and analytic results}

The frequency of inspection visits carried out by government district drug inspectors in both the intervention and comparison districts of the study are presented in table 2. Following the introduction of peer supervision, the mean (SD) monthly visits for district drug inspectors in the intervention district was $0.98(0.34)$ while in the comparison district was $0.78(0.31)$. The mean (SD) visits for peer supervisors in the intervention district was $1.2(0.87)$. 
The aggregated monthly district percentages of appropriately treated children are presented in table 3 and Fig.2. There was a general increase in the overall percentage of children treated appropriately for pneumonia symptoms (using amoxicillin tablets) and non-bloody diarrhoea (using a combination of zinc and oral rehydration salts) in both districts months preceding introduction of peer supervision.

Following the introduction of peer supervision, there were monthly improvements in the overall appropriate treatment of children with pneumonia symptoms in the intervention district while there was a decline in the comparison district.

There was no difference in appropriate treatment of children with uncomplicated malaria between the two districts. Noticeably, more children were appropriately treated for non-bloody diarrhoea than malaria and pneumonia symptoms in both districts over the study period.

\section{Effectiveness of peer supervision on appropriate treatment of Pneumonia symptoms}

As shown in regression table 4 and visual inspection of Fig. 3, prior to introduction of peer supervision, 21.4\% of the children were appropriately treated for pneumonia symptoms in the comparison district. This was followed by a $0.37 \%$ monthly decrease $(\mathrm{P}=0.59, \mathrm{CI}=[-1.80,1.06])$ until October month after introduction of peer supervision (November 2016), the proportion of children appropriately treated for pneumonia symptoms was $10.84 \%$ significantly higher in the intervention $(\mathrm{P}<0.05, \mathrm{CI}=[1.75,19.9])$ compared to the comparison district.

This was followed by a decrease in appropriate treatment of children with pneumonia symptoms of $2.19 \%$ per month $(\mathrm{P}=0.07, \mathrm{CI}=[-4.56,0.18])$. The intervention decreased the proportion of appropriately treated children with pneumonia symptoms by $1.68 \%$ during the intervention period $(\mathrm{p}=0.29, \mathrm{CI}=[-4.93$, 1.57]) compared to the comparison district. Post intervention trend results revealed that introduction of the intervention increased the proportion of appropriately treated children exhibiting pneumonia symptoms at a rate of $1.21 \%(\mathrm{p}=0.008, \mathrm{CI}=[0.36,2.05])$

Effectiveness of peer supervision on appropriate treatment of uncomplicated Malaria

Again, from regression table 4 and visual inspection of Fig. 4, prior to introduction of peer supervision, $50.6 \%$ of the children were appropriately treated for uncomplicated malaria in the comparison district. Then, a $0.44 \%$ monthly increase $(\mathrm{P}=0.585, \mathrm{CI}=[-1.23,2.11])$ occurred until October 2016.

A month after introduction of peer supervision (November 2016), the proportion of appropriately treated children for uncomplicated malaria was $1.46 \%$ higher in the intervention $(\mathrm{P}=0.79, \mathrm{CI}=[-10.43,13.36])$ compared to the comparison district. However, this difference was not statistically significant. This was followed by a small increase in appropriate uncomplicated malaria treatment of $0.19 \%$ per month $(\mathrm{P}=$ $0.88, \mathrm{CI}=[-2.39,2.77])$. The intervention decreased the proportion of children appropriately treated for uncomplicated malaria by $1.98 \%$ during the intervention period $(\mathrm{p}=0.38, \mathrm{CI}=[-6.60,2.63])$ compared to the comparison district. In addition, post intervention trend results revealed that introduction of the intervention increased the proportion of appropriately treated children with uncomplicated malaria at a rate of $1.14 \%(\mathrm{p}=0.34, \mathrm{CI}=[-1.30,3.58])$

\section{Effectiveness of peer supervision on appropriate treatment of non-bloody diarrhoea}

As seen in regression table 4 and visual inspection of Fig. 5, prior to introduction of the intervention (peer supervision), the proportion of appropriately treated children with non-bloody diarrhoea in the comparison district was $51.3 \%$. This was followed by a $2 \%$ monthly increase $(\mathrm{P}=0.099, \mathrm{CI}=[-1.32,5.44])$ until October 2016.

In the first month of the intervention (November 2016), the proportion of appropriately treated children with non-bloody diarrhoea was $4 \%$ higher in the intervention $(\mathrm{P}=0.448, \mathrm{CI}=[-9.85,17.19])$ compared to the comparison district. However, this difference was not statistically significant. Consequently, there was a slight decrease in appropriate non-bloody diarrhoea treatment of $0.37 \%$ per month $(\mathrm{P}=0.74, \mathrm{CI}=$ $[-3.74,2.99])$. The intervention significantly decreased the proportion of children appropriately treated for 
non-bloody diarrhoea by $4 \%$ during the intervention period ( $\mathrm{p}<0.05, \mathrm{CI}=[-7.95,-0.13])$ compared to the comparison district. In addition, post intervention trend results revealed that introduction of the intervention decreased the proportion of appropriately treated children at a rate of $1 \%(\mathrm{p}<0.06, \mathrm{CI}=[-1.95,0.02])$.

\section{Discussion}

This study aimed at assessing the effectiveness of peer supervision on paediatric fever treatment among registered private drug sellers in two districts in East-Central Uganda. There was an increase in the number of inspection visits by the district drug inspector in the intervention district after peer-supervision was introduced. Overall, post intervention trend results revealed that introduction of peer supervision was associated with a significant long term increase in the proportion of appropriately treated children with pneumonia symptoms. Peer supervision did not increase the proportion of appropriately treated children with uncomplicated malaria and non-bloody diarrhoea.

\section{Government inspection and peer supervision visits}

Government recommends monthly inspection visits by district drug inspectors. However, results in table 3 revealed that inspection was not done as per statutory recommendations. The slight but insignificant increase in number of inspection visits by the district drug inspector in the intervention district were in tandem with what has been referred to by many scholars as social proof ${ }^{34,35}$. In the social proof theory, people tend to do things because other people are doing them. This is in line with what the researchers of this study wanted to achieve-strengthening the district health system. Given that there was no statistical difference in inspection visits between DDIs from the two districts overall, we were able to attribute observable intervention effects to peer supervision.

\section{Effectiveness of peer supervision on appropriate treatment of pneumonia symptoms}

Examination of health system effects due to peer supervision at district level revealed that appropriate treatment of pneumonia symptoms using antibiotics may be achieved on a long term rather than short term basis. These results are in agreement with those obtained by Li Zhen and colleagues while looking at the impact of prescription audit and feedback for antibiotic use in rural clinics ${ }^{36}$. The difference is that while Li Zhen and colleagues used a single group comparison, the investigators of this study used a reference group which may have strengthened the validity of the findings. Evidence from different settings shows that even among trained community health workers, diagnosis and rightful classification of children

with pneumonia symptoms remains a challenge ${ }^{37-39}$. As such, several studies emphasize the importance of supervision in improving appropriate treatment of acute respiratory tract infections which are associated with community pneumonia in children under five years of age ${ }^{40,41}$. This further strengthens the importance of peer supervision among drug sellers in rural settings where supervision has been inadequate. Thus, even though drug sellers in both districts were trained prior to introduction of peer supervision, the continuous professional development received through peer supervision improved diagnosis of pneumonia symptoms

\section{Effectiveness of peer supervision on appropriate treatment of uncomplicated Malaria}

Considering results one month after introduction of peer supervision as well as post trend results, there was no statistically significant difference in appropriate treatment of uncomplicated malaria among drug sellers in the intervention and comparison districts. In Uganda, studies evaluating integrated community case management in eight districts of central Uganda also found no statistical difference in appropriate treatment of children between intervention and comparison districts ${ }^{41}$. This may be because malaria has affected the Ugandan population for a long time. As such, many drug sellers are able to treat uncomplicated malaria even without formal training and or supervision.

\section{Effectiveness of peer supervision on appropriate treatment of non-bloody diarrhoea}

The results show that peer supervision did not improve appropriate non-bloody diarrhoea treatment in children in the intervention district compared to the comparison district. 
This is because in both districts, drug sellers prescribed more ORS and not Zinc and yet they should have prescribed the two as a combination. These study results are in agreement with what Agnes Nanyonjo et al., found while seeking to understand the impact of the iCCM strategy on uptake of appropriate diarrhoea and pneumonia treatment in Uganda ${ }^{42}$. In her study, she points to the fact that this behaviour is driven more by poverty levels of the population. The East-Central region is one of the poorest regions in Uganda ${ }^{43}$. Nevertheless, there is need to discourage the sale of zinc and ORS as separate entities because this seems to hype inappropriate treatment of non-bloody diarrhoea. Drug sellers need to be encouraged to stock and prescribe co-packaged ORS and zinc since evidence shows that a combination improves adherence to and appropriate treatment of non-bloody diarrhoea in children under five years of age ${ }^{44,45}$.

Study limitations

In order to obtain valid results for all the three outcomes (pneumonia symptoms, uncomplicated malaria and non-bloody diarrhoea), the investigators ought to have found a district that was comparable to Luuka (the intervention district) both at the baseline average $(\mathrm{Z})$ and the baseline trend $(\mathrm{ZTt}$ ) before peer supervision was introduced. However, baseline average and trend was done for uncomplicated malaria only. This may have affected the results presented in this paper.

Appropriate treatment of children less than five years with symptoms of pneumonia, uncomplicated malaria and non-bloody diarrhoea is done with reference to the child's age. In many rural settings, a record of the child's birth date is not accurately recorded. Therefore the investigators of this study feel that since appropriate treatment is based on age, this may have affected the results.

\section{Policy implications}

The national professional guidelines for licensing, renewal and new licenses for class $\mathrm{C}$ drug shops in Uganda stipulate that licensed drug sellers are responsible for supervising drug shops ${ }^{16}$. This may be one of the reasons contributing to persistent inappropriate treatment of children less than five years of age by drug sellers since their primary business motive is profit. Peer supervision may be an effective method of improving appropriate treatment of children aged five years or less with febrile illnesses.

Areas for more research

The researchers feel that while effectiveness of peer supervision on appropriate treatment among drug sellers has been assessed at aggregate district level, it is still not clear whether prescribed drugs are given to children in right doses by care givers after they have been dispensed by drug sellers. There is need for more research to understand what happens at household level.

\section{Conclusions}

In conclusion, this study demonstrated that peer supervision was associated with a significant long term increase in appropriate treatment of children aged five years or less with pneumonia symptoms but not uncomplicated malaria and non-bloody diarrhoea. Implementation of community level interventions to improve paediatric fever management should consider embedding the component of peer-supervision.

Acknowledgements

We are grateful to all the data collectors, drug shop operators, peer supervisors and district health officers of the participating districts. We appreciate all the help we obtained from Makerere University, Uppsala University, UNICEF, University of Trent and the Erasmus+ mobility programme. Funding for this study was obtained from Uppsala University and the Einhorn family foundation. Staff from Uppsala University helped with interpretation of the data and writing the manuscript.

Competing interests

The authors declare that they have no competing interests.

Ethics approval and consent to participate 
The Higher Degrees, Research and Ethics Committee (HDREC) of the school of public health, college of health sciences, Makerere University Kampala and the Uganda National council of Science and technology (SS4703) approved the study protocol. All drug sellers provided written informed consent for themselves and for the children whose information was abstracted from sick child registers.

Authorship

SP, HW, AM and $\mathrm{AB}$ conceived and designed the experiment. AB, HW and MM implemented the study idea including the supervision of data collection. AB, MM, PA, FK, DM participated in data analysis. All authors proof read, the original draft, helped in refining ideas that led to the final manuscript.

Data Storage and Documentation

Data sets used during the study are available from the corresponding author on reasonable request

\section{References}

1. Morris SK, Bassani DG, Awasthi S, et al. Diarrhea, Pneumonia, and Infectious Disease Mortality in Children Aged 5 to 14 Years in India. PLOS ONE. 2011;6(5):e20119.

2. Johri M, Ridde V, Heinmuller R, Haddad S. Estimation of maternal and child mortality one year after user-fee elimination: an impact evaluation and modelling study in Burkina Faso. Bulletin of the World Health Organization. Oct 1 2014;92(10):706-715.

3. Nsabagasani X, Ogwal-Okeng J, Mbonye A, Ssengooba F, Muhumuza S, Hansen EH. Availability and utilization of the WHO recommended priority lifesaving medicines for under five-year old children in public health facilities in Uganda: a cross-sectional survey. Journal of pharmaceutical policy and practice. 2015;8(1):18.

4. WHO. Diarrhoeal disease-Fact sheet. World Health Organisation;2017.

5. WHO. World malaria report 2019. Geneva: World Health Organisation;2019.

6. WHO. Pneumonia-Fact sheetGeneva: World Health Organisation 2019.

7. WHO. World Malaria Report.Geneva: World Health Organisation;2018.

8. UNICEF. One is too many: Ending child deaths from pneumonia and diarrhea. New York: UNICEF;2016.

9. MoH. ANNUAL HEALTH SECTOR PERFROMANCE REPORT. Kampala,Uganda: Ministry of Health;2019.

10. Young M, Wolfheim C, Marsh DR, Hammamy D. World Health Organization/United Nations Children's Fund joint statement on integrated community case management: an equity-focused strategy to improve access to essential treatment services for children. The American journal of tropical medicine and hygiene. Nov 2012;87(5 Suppl):6-10.

11. WHO, UNICEF. WHO/UNICEF JOINT STATEMENT: Integrated Community Case Management (iCCM). Geneva 2012.

12. Mayora C, Kitutu FE, Kandala N-B, Ekirapa-Kiracho E, Peterson SS, Wamani H. Private retail drug shops: what they are, how they operate, and implications for health care delivery in rural Uganda. $B M C$ Health Serv Res. 2018/07/09 2018;18(1):532.

13. Konde-Lule J, Gitta SN, Lindfors A, Okuonzi S, Onama VON, Forsberg BC. Private and public health care in rural areas of Uganda. BMC International Health and Human Rights.2010/11/24 2010;10(1):29.

14. NMCD, UBOS, ICF. Uganda Malaria Indicator Survey 2018-19. Kampala, Uganda, and Rockville, Maryland, USA: Ministry of Health;2020. 
15. Awor P, Wamani H, Tylleskar T, Peterson S. Drug seller adherence to clinical protocols with integrated management of malaria, pneumonia and diarrhoea at drug shops in Uganda.Malar J. Jul 16 2015;14:277.

16. NDA. Professional guidelines for licensing,renewal and new licenses for class $\mathrm{C}$ drug shops in Uganda. National Drug Authority; 2018.

17. HSC. Guidelines for Recruitment of Health Workers in Districs and Urban Authorities 2005.Kampala: Health Service Comission;2005.

18. Briesacher BA, Soumerai SB, Zhang F, et al. A critical review of methods to evaluate the impact of FDA regulatory actions. Pharmacoepidemiology and drug safety. Sep 2013;22(9):986-994.

19. Harris AD, McGregor JC, Perencevich EN, et al. The use and interpretation of quasi-experimental studies in medical informatics. Journal of the American Medical Informatics Association : JAMIA. Jan-Feb 2006;13(1):16-23.

20. Penfold RB, Zhang F. Use of interrupted time series analysis in evaluating health care quality improvements. Academic pediatrics. Nov-Dec 2013;13(6 Suppl):S38-44.

21. Avortri GS, Nabukalu JB, Nabyonga-Orem J. Supportive supervision to improve service delivery in low-income countries: is there a conceptual problem or a strategy problem? BMJ Glob Health. 2019;4(Suppl 9):e001151-e001151.

22. Boniface G, Seymour A, Polglase T, Lawrie C, Clarke M. Exploring the Nature of Peer and Academic Supervision on a Role-Emerging Placement. British Journal of Occupational Therapy. 2012/04/01 2012;75(4):196-201.

23. Abdulrahman SA, Rampal L, Ibrahim F, Radhakrishnan AP, Kadir Shahar H, Othman N. Mobile phone reminders and peer counseling improve adherence and treatment outcomes of patients on ART in Malaysia: A randomized clinical trial. PLOS ONE.2017;12(5):e0177698.

24. Engebretsen IM, Nankabirwa V, Doherty T, et al. Early infant feeding practices in three African countries: the PROMISE-EBF trial promoting exclusive breastfeeding by peer counsellors. International breastfeeding journal. 2014;9:19.

25. Gatlin TK, Serafica R. Systematic review of peer education intervention programmes among individuals with type 2 diabetes. Dec 2017;26(23-24):4212-4222.

26. UBOS. The National Population and Housing Census 2014 - Area Specific Profile Series. Kampala, Uganda.: Uganda Bureau of Statistics 2017.

27. UNICEF, WHO, MoH.Integrated Community Case Management of Childhood Malaria,Pneumonia and Diarrhoea: Implementation Guidelines. Kampala, Uganda: Ministry of Health, Kampala, Uganda;2010.

28. UBOS. The National Population and Housing Census 2014 - Health status and

Associated factors. Kampala, Uganda: Uganda Bureau of Statistics;2017.

29. Linden A. Conducting Interrupted Time-series Analysis for Single- and Multiple-group Comparisons. The Stata Journal. 2015;15(2):480-500.

30. Hudson J, Fielding S, Ramsay CR. Methodology and reporting characteristics of studies using interrupted time series design in healthcare. BMC Medical Research Methodology. 2019/07/04 2019;19(1):137.

31. Stoker LJ, Heerdink ER, Janssen R, Egberts TCG. Effect of reimbursement restriction policy on the use of benzodiazepines in the Netherlands: an interrupted time series analysis.BMJ Open. 2019;9(9):e029148.

32. Bernal JL, Cummins S, Gasparrini A. Interrupted time series regression for the evaluation of public health interventions: a tutorial. International Journal of Epidemiology. 2016;46(1):348-355. 
33. Fretheim A, Zhang F, Ross-Degnan D, et al. A reanalysis of cluster randomized trials showed interrupted time-series studies were valuable in health system evaluation. Journal of Clinical Epidemiology. 2015/03/01/ 2015;68(3):324-333.

34. Facci EL, Bell MA, Nayeem R. The Effect of Social Proof on Weather-Related Decision Making in Aviation.Proceedings of the Human Factors and Ergonomics Society Annual Meeting. 2005/09/01 2005;49(19):1780-1784.

35. Hilverda F, Kuttschreuter M, Giebels E. The Effect of Online Social Proof Regarding Organic Food: Comments and Likes on Facebook. Frontiers in Communication.2018-August-03 2018;3(30).

36. Zhen L, Jin C, Xu H-N. The impact of prescriptions audit and feedback for antibiotic use in rural clinics: interrupted time series with segmented regression analysis. BMC health services research. 2018;18(1):777777.

37. Miller NP, Amouzou A, Tafesse M, et al. Integrated community case management of childhood illness in Ethiopia: implementation strength and quality of care. The American journal of tropical medicine and hygiene. Aug 2014;91(2):424-434.

38. Mukanga D, Babirye R, Peterson S, et al. Can lay community health workers be trained to use diagnostics to distinguish and treat malaria and pneumonia in children? Lessons from rural Uganda. Tropical medicine E international health : TM \& IH. Oct 2011;16(10):1234-1242.

39. Noordam AC, Barbera Lainez Y, Sadruddin S, et al. The use of counting beads to improve the classification of fast breathing in low-resource settings: a multi-country review. Health Policy Plan. Jul 2015;30(6):696-704.

40. Addo-Yobo E, Anh DD, El-Sayed HF, et al. Outpatient treatment of children with severe pneumonia with oral amoxicillin in four countries: the MASS study. Tropical Medicine 8 International Health. 2011;16(8):995-1006.

41. Mubiru D, Byabasheija R, Bwanika JB, et al. Evaluation of Integrated Community Case Management in Eight Districts of Central Uganda. PloS one.2015;10(8):e0134767-e0134767.

42. Nanyonjo A, Ssekitooleko J, Counihan H, Makumbi F, Tomson G, Källander K. Impact of an integrated community case management programme on uptake of appropriate diarrhoea and pneumonia treatments in Uganda: A propensity score matching and equity analysis study. Int J Equity Health. 2015;14:74-74.

43. Rafa M, Moyer J, Sutton XWaP.Estimating District GDP in Uganda. USAID;2017.

44. Habib MA, Soofi S, Sadiq K, et al. A study to evaluate the acceptability, feasibility and impact of packaged interventions ("Diarrhea Pack") for prevention and treatment of childhood diarrhea in rural Pakistan. BMC Public Health. Oct 3 2013;13:922.

45. Kitutu FE, Kalyango JN, Mayora C, Selling KE, Peterson S, Wamani H. Integrated community case management by drug sellers influences appropriate treatment of paediatric febrile illness in South Western Uganda: a quasi-experimental study. Malaria Journal. 2017/10/23 2017;16(1):425.

Table 1: Definitions of variable names used in the econometric analysis

\begin{tabular}{ll}
\hline Variable names & Definitions \\
\hline $\mathrm{y}_{\mathrm{t}}$ & Aggregated outcome variable (appropriately drug use in treatment of malaria, diarrhoea and $\mathrm{p}$ \\
$\beta_{\mathrm{o}}$ & Intercept which represents the starting level of the outcome variables (appropriately drug use $\mathrm{i}$ \\
$\mathrm{T}_{\mathrm{t}}$ & Equally spaced time points in months \\
$\mathrm{X}_{\mathrm{t}}$ & Indicator variable representing pre-peer supervision period-0, otherwise 1 \\
$\mathrm{Z}$ & Indicator variable representing intervention and comparison groups (Luuka-1,Buyende -0) \\
$\mathrm{X}_{\mathrm{t}} \mathrm{T}_{\mathrm{t}}, \mathrm{ZT}_{\mathrm{t}}, \mathrm{ZX}_{\mathrm{t}}$ and $\mathrm{ZX}_{\mathrm{t}} \mathrm{T}_{\mathrm{t}}$ & Interaction terms
\end{tabular}




\begin{tabular}{ll}
\hline Variable names & Definitions \\
\hline$\beta_{1}$ & Slope or trajectory of the outcome variable (appropriately drug use) until the introduction of $p$ \\
$\beta_{2}$ & Change in the level of appropriately drug use that occurs in the period immediately following t \\
$\beta_{3}$ & Difference between pre-peer supervision and post-peer supervision slopes of the Outcome \\
$\beta_{4}$ & Difference in the level between intervention and comparison prior to peer supervision \\
$\beta_{5}$ & Difference in the slope between intervention and comparison prior to peer supervision \\
$\beta_{6}$ & Difference in level between intervention and comparison in the period immediately following int \\
$\beta_{7}$ & Difference between intervention and comparison in the slope after initiation of peer supervision \\
$\varepsilon_{\mathrm{t}}$ & Error term at time t representing unexplained random variability in the model \\
\hline
\end{tabular}

Table 2: Frequency of inspection and peer supervision visits in the study period

\begin{tabular}{lll}
\hline Number of visits & Intervention (Luuka) & Comparison(Buyende) \\
\hline Inspection visits before introduction of peer supervisors & & \\
Median & 0.88 & 1.00 \\
Mean & 0.88 & 0.94 \\
std. deviation & 0.31 & 0.38 \\
Inspection visits following introduction of peer supervisors & & \\
Median & 1.00 & 0.86 \\
Mean & 0.98 & 0.78 \\
std. deviation & 0.34 & 0.31 \\
Peer supervision visits & 1.00 \\
Median & 1.2 & - \\
Mean & 0.87 & - \\
std. deviation & & \\
\hline
\end{tabular}

Table 3: Monthly appropriate treatment of childhood illnesses by district (\%)

\begin{tabular}{|c|c|c|c|c|c|}
\hline \multicolumn{2}{|l|}{ District } & \multirow{2}{*}{\begin{tabular}{l|} 
Month \\
$16-J u n$
\end{tabular}} & \multirow{2}{*}{$\begin{array}{l}\text { Appropriate diarrhoea treatment } \\
57.3\end{array}$} & \multirow{2}{*}{$\begin{array}{l}\text { Appropriate Malaria treatment } \\
45.8\end{array}$} & \multirow{2}{*}{$\begin{array}{l}\text { Appro } \\
11.2\end{array}$} \\
\hline Intervention & Before & & & & \\
\hline & & 16-Jul & 70.1 & 60.4 & 26 \\
\hline & & 16-Aug & 63.3 & 53.2 & 27.9 \\
\hline & & 16-Sep & 73.8 & 56.8 & 26 \\
\hline & & 16-Oct & 72.7 & 62.3 & 36.6 \\
\hline & After & $16-\mathrm{Nov}$ & 75.1 & 66.3 & 36.9 \\
\hline & & 16-Dec & 74.1 & 53.5 & 30.5 \\
\hline & & 17-Jan & 68.1 & 56.5 & 37.8 \\
\hline & & 17-Feb & 74 & 64.1 & 41.6 \\
\hline & & 17-Mar & 64.6 & 63.8 & 42 \\
\hline & & 17-Apr & 69.9 & 66 & 37.8 \\
\hline & & 17-May & 70.1 & 66.2 & 41.9 \\
\hline \multirow[t]{7}{*}{ Comparison } & Before & 16-Jun & 48.3 & 51.8 & 22.9 \\
\hline & & 16-Jul & 65.7 & 50.6 & 21.5 \\
\hline & & 16-Aug & 44 & 47.1 & 17.9 \\
\hline & & 16-Sep & 55.5 & 57 & 18.4 \\
\hline & & 16-Oct & 63.7 & 50.8 & 22.6 \\
\hline & After & $16-\mathrm{Nov}$ & 63 & 49.5 & 24.8 \\
\hline & & 16-Dec & 67.9 & 51.8 & 33.9 \\
\hline
\end{tabular}




\begin{tabular}{lllll}
\hline District & Month & Appropriate diarrhoea treatment & Appropriate Malaria treatment & Appro \\
\hline & 17-Jan & 65 & 62.6 & 19.7 \\
& 17-Feb & 84.6 & 67.1 & 24 \\
17-Mar & 63 & 47.1 & 32 \\
& 17-Apr & 72.9 & 59.2 & 15.4 \\
& 17-May & 76.1 & 55.6 & 9.1 \\
\hline
\end{tabular}

Table 4: Effect of peer supervision on appropriate treatment of childhood illnesses

\begin{tabular}{ll}
\hline Variable & Diarrhoea \\
\hline & Coef \\
Time $(\mathrm{T})$ & 2.06 \\
Baseline slope $(\mathrm{Z})$ & 9.22 \\
Interaction $\left(\mathrm{Z}^{*} \mathrm{~T}\right)$ & 1.39 \\
Pre-intervention slope $(\mathrm{X})$ & 3.67 \\
Interaction $\left(\mathrm{X}^{*} \mathrm{~T}\right)$ & -0.37 \\
Intervention slope $\left(\mathrm{Z}^{*} \mathrm{X}\right)$ & -7.73 \\
Interaction $\left(\mathrm{Z}^{*} \mathrm{X}\right.$ T) + & -4.04 \\
Constant ++ & 51.3 \\
Post intervention trend (November 2016 to May 2017) & Post intervention trend (November 2016 to May 2 \\
Intervention & -0.96 \\
Comparison & 1.69 \\
\hline
\end{tabular}

+ Difference between pre-intervention and post-intervention slope (effect of the intervention over time)

++ Represents the starting level or percentage of infants appropriately treated in the comparison district.

$* P<.05$

$* * P<.001$

\section{Hosted file}

image1.emf available at https://authorea.com/users/334671/articles/460639-effectiveness-ofpeer-supervision-on-paediatric-fever-treatment-among-registered-private-drug-sellers-ineast-central-uganda-an-interrupted-time-series-analysis

Fig. 2: Trend in month-to-month appropriate treatment of childhood illnesses June 2016 to May 2017

\section{Hosted file}

image2.emf available at https://authorea.com/users/334671/articles/460639-effectiveness-ofpeer-supervision-on-paediatric-fever-treatment-among-registered-private-drug-sellers-ineast-central-uganda-an-interrupted-time-series-analysis

Fig. 3: Newey-West regression analysis for monthly rational treatment of pneumonia, June 2016 to May 2017

\section{Hosted file}

image3.emf available at https://authorea.com/users/334671/articles/460639-effectiveness-ofpeer-supervision-on-paediatric-fever-treatment-among-registered-private-drug-sellers-ineast-central-uganda-an-interrupted-time-series-analysis 
Fig. 4: Newey-West regression analysis for monthly rational treatment of malaria, June 2016 to May 2017

\section{Hosted file}

image4.emf available at https://authorea.com/users/334671/articles/460639-effectiveness-ofpeer-supervision-on-paediatric-fever-treatment-among-registered-private-drug-sellers-ineast-central-uganda-an-interrupted-time-series-analysis

Fig. 5: Newey-West regression analysis for monthly rational treatment of diarrhoea, June 2016 to May 2017

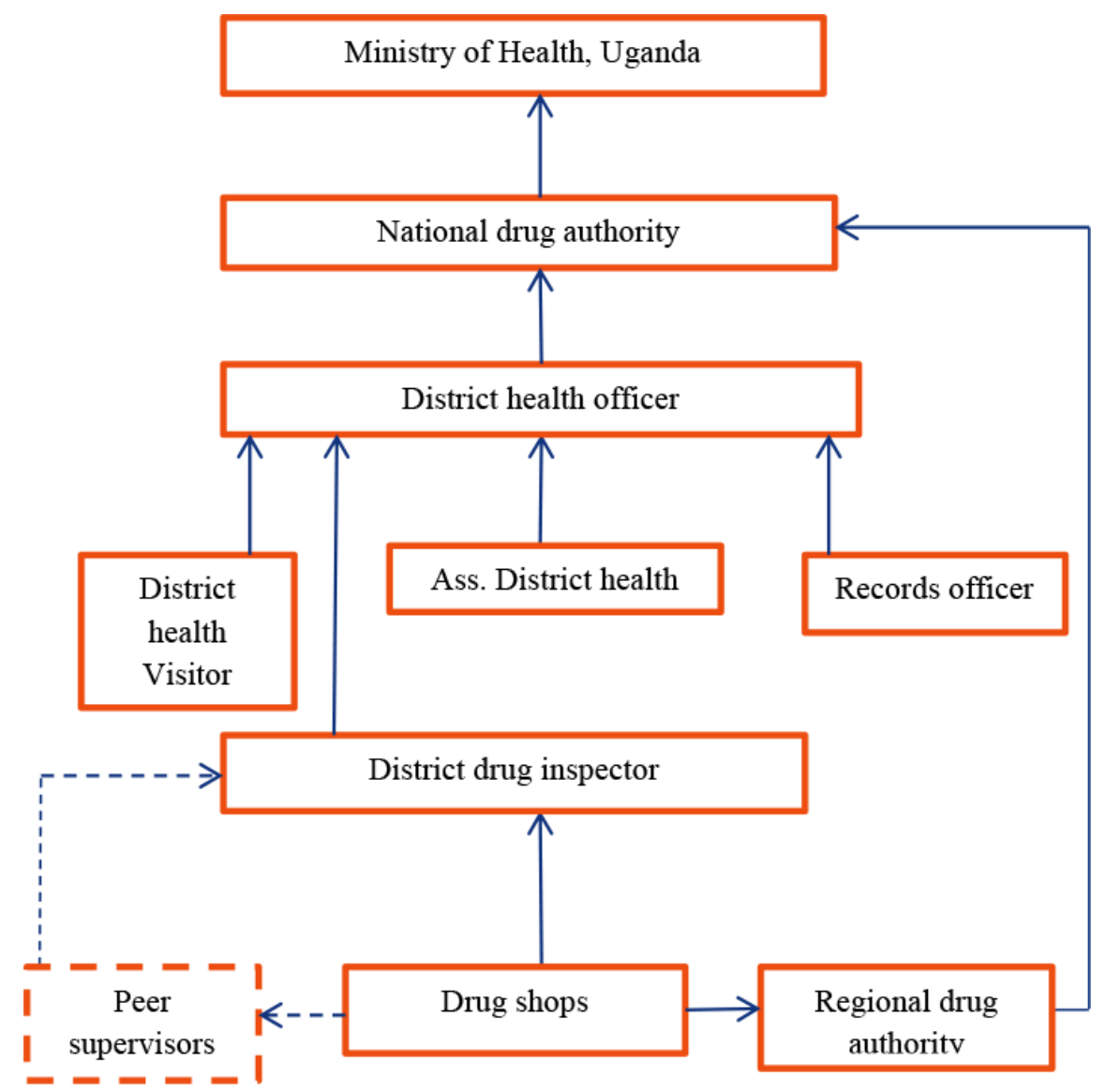

Fig. 1: The peer supervision process 


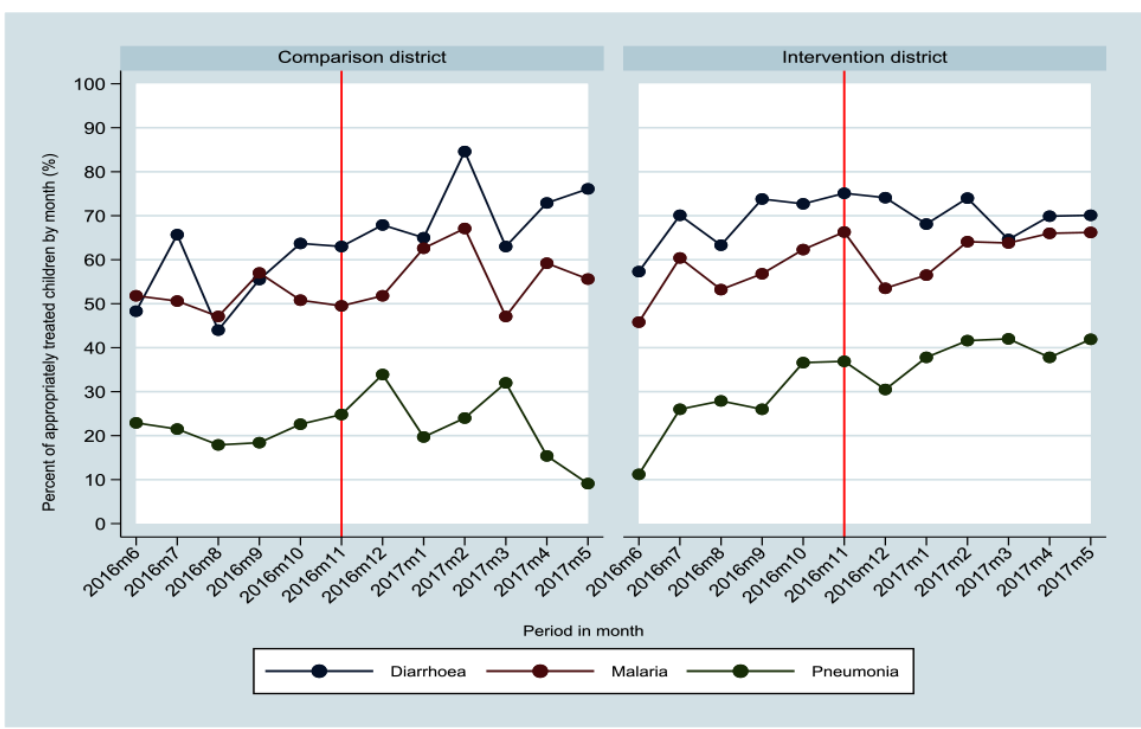

Fig. 1: Trend in month-to-month appropriate treatment of childhood illnesses June 2016 to May 2017

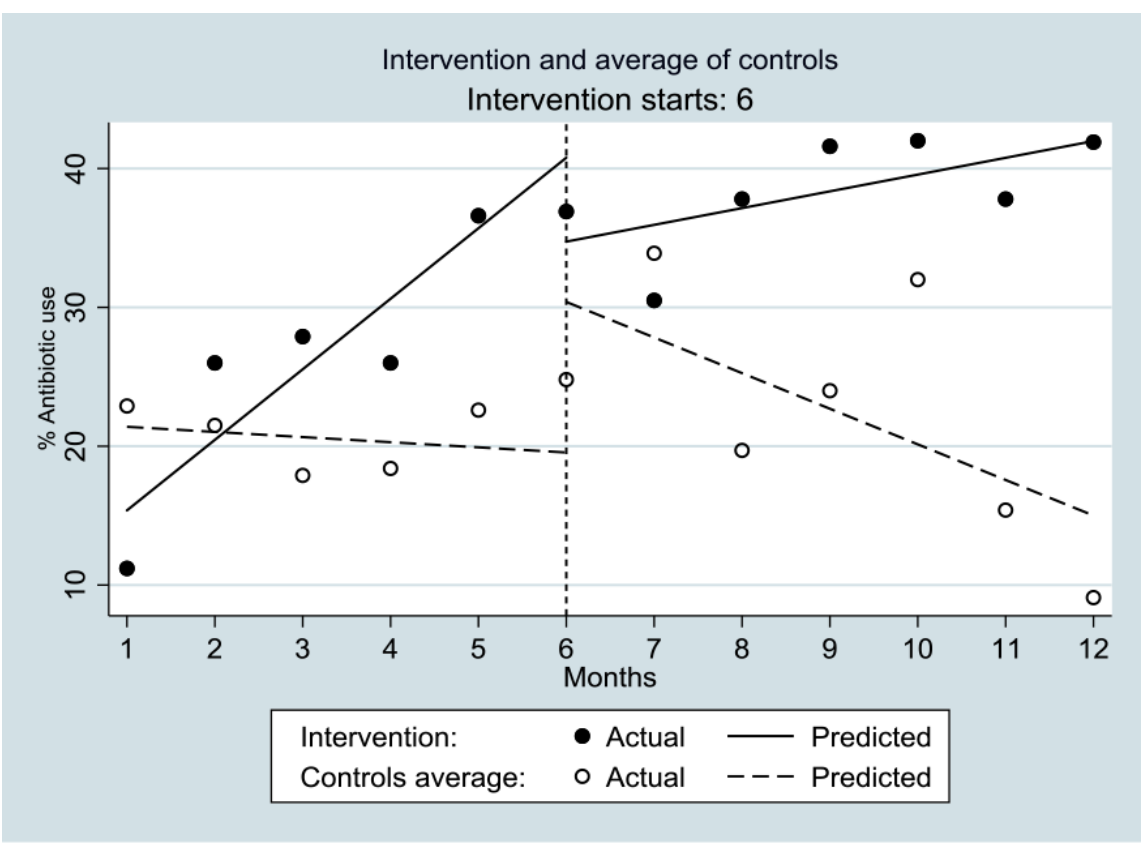



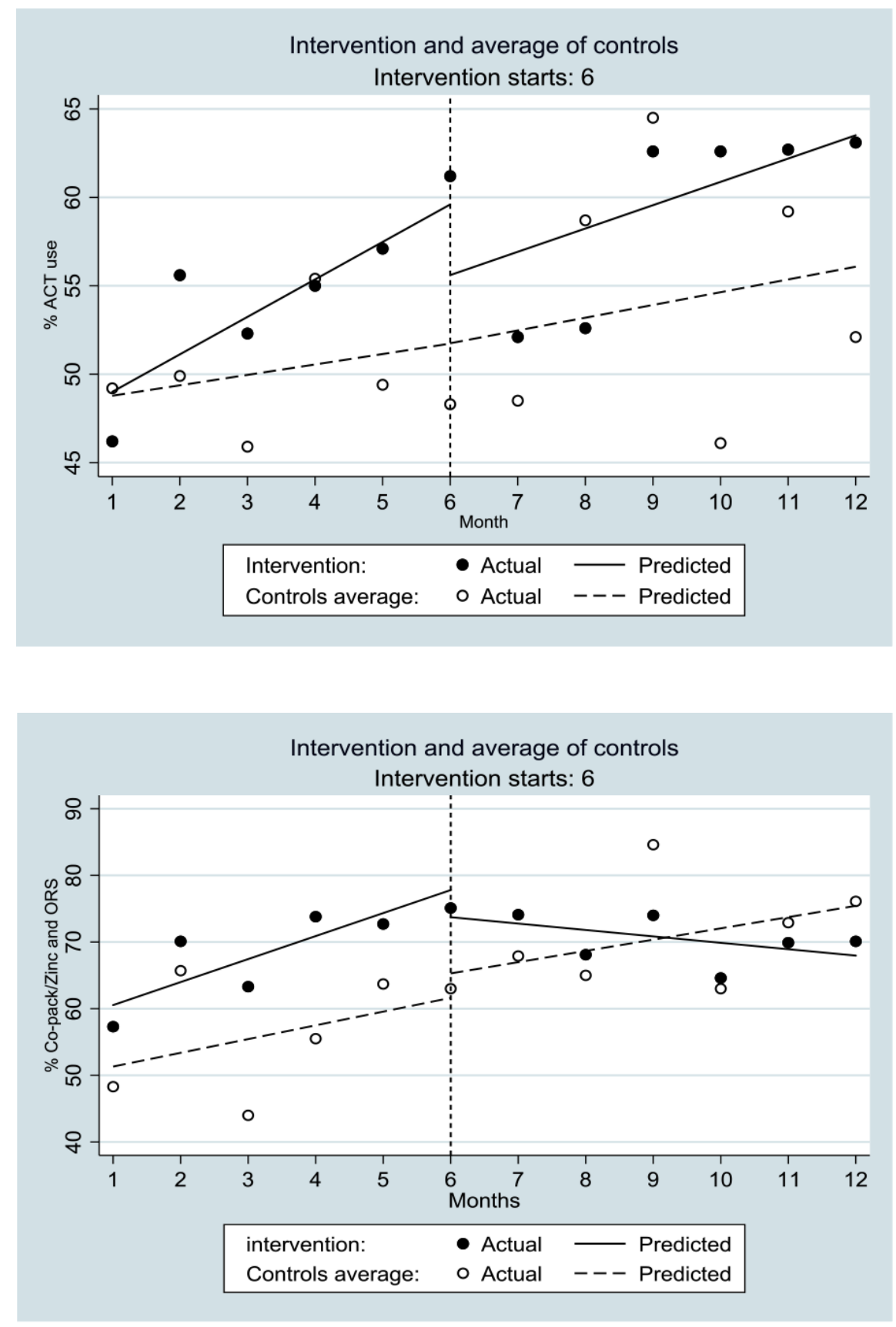

Fig. 1: Newey-West regression analysis for monthly rational treatment of diarrhoea, June 2016 to May 2017 